\title{
PARTIALLY ADAPTIVE TRANSMIT BEAMFORMING FOR SEARCH FREE 2D DOA ESTIMATION IN MIMO RADAR
}

\author{
Matthew W. Morency and Sergiy A. Vorobyov \\ Department of Signal Processing and Acoustics, Aalto University, Finland \\ Email: matthew.morency@aalto.fi, sergiy.vorobyov@aalto.fi
}

\begin{abstract}
In this paper, a partially adaptive two dimensional (2D) transmit beamforming approach is proposed to enable search-free azimuth and elevation direction of arrival (DOA) estimation in MIMO radar. Specifically, the 2D transmit array is nonadaptively partitioned into a number of subarrays. Then, a beamspace matrix is adaptively designed for each subarray, such that the beampatterns corresponding to each matrix have the exact same magnitude. By constraining the beams to be transmitted from different subarrays, multiple data invariances are enforced independently of the receive array geometry. The invariances are then exploited by search-free DOA estimation methods. Simulations validate the proposed approach.
\end{abstract}

Index Terms - Adaptive beamforming, MIMO radar, search-free DOA estimation methods

\section{INTRODUCTION}

Under the concept of MIMO radar [1]- [3] a variety of questions on the topics ranging from waveform design and selection [5], to spatial configurations of radar systems [3], [6]- [9], and transmit beamforming design [8]- [11] have been investigated. Recently, several transmit beamforming approaches have been proposed to allow the use of low-complexity search-free direction of arrival (DOA) methods both in one and two dimensions (2D), while relaxing requirements on array geometries [12]- [14]. There is substantial motivation for transmit beamforming with reduced waveform diversity. Firstly, it was shown in [9] that the performance of a MIMO radar system which uses less than full waveform diversity and provides transmit pre-processing gain is superior to that of a MIMO radar system which uses full waveform diversity and provides no pre-processing gain. Further, as the number of antenna elements in 2D transmit arrays can be very large, full waveform diversity presents significant issues including increased computational complexity at the receiver.

In this paper, a 2D transmit beamformer for planar arrays is designed with the specific goal of creating a data set at the receive array with multiple invariances independently of the receive array geometry. This is altogether a different scenario than the one investigated in [14], but with the same goal in mind. To do this, we use a partially adaptive approach: that is, the optimization is constrained to only consider a subset of transmit antenna elements. A simple transformation is introduced which creates a set of identical beams which are transmitted from different subarrays. Since each beam has the same beampattern, but originates from a linearly displaced subarray, the data which is collected from each beam is related to that of the other beams through a phase rotation which depends directly on the target location. Therefore, we can use all beams to estimate both the elevation and azimuth of a target using search-free DOA estimation methods.

\section{SYSTEM MODEL}

Consider a monostatic MIMO radar system consisting of a uniform rectangular array (URA) with $P \times Q$ antenna elements acting as a transmitter, and a planar receive array with $R$ antenna elements in an arbitrary configuration. The $P Q \times 1$ transmit array response vector is defined as

$$
\mathbf{a}(\theta, \phi)=\operatorname{vec}\left(\mathbf{u}(\theta, \phi) \mathbf{v}^{T}(\theta, \phi)\right)
$$

where $[\mathbf{u}(\theta, \phi)]_{p}=e^{j 2 \pi p \cdot d_{x} \sin \theta \cos \phi}, p \in\{0,1, \cdots, P-1\}$ and $[\mathbf{v}(\theta, \phi)]_{q}=e^{j 2 \pi q \cdot d_{y} \sin \theta \sin \phi}, q \in\{0,1, \cdots, Q-1\}$ correspond to the antenna response coefficients for displacements $p \cdot d_{x}$ and $q \cdot d_{y}$ from a reference element respectively, and $(\cdot)^{T}$ is the vector transpose operator. A linear combination of $K$ orthogonal baseband waveforms $\psi(t)=$ $\left[\psi_{1}(t), \ldots, \psi_{K}(t)\right]^{T}$ is transmitted, where $K \ll P Q$, which permits energy focussing over a desired sector $\Theta=\left[\begin{array}{ll}\theta_{1} & \theta_{2}\end{array}\right]$ in the elevation domain and $\Phi=\left[\begin{array}{ll}\phi_{1} & \phi_{2}\end{array}\right]$ in the azimuthal domain.

The signal at the transmitter within a single slow-time pulse, for a given angular direction $(\theta, \phi), \theta \in\left[-\frac{\pi}{2}, \frac{\pi}{2}\right]$ and $\phi \in[0,2 \pi]$, at time $t$ is expressed as

$$
\mathbf{s}(t)=\mathbf{a}(\theta, \phi)^{H} \mathbf{W} \boldsymbol{\psi}(t)
$$

where $\mathbf{W}$ is a $P Q \times K$ complex valued beamforming weight matrix with $w_{n, k}$ corresponding to the weighting coefficient 
of waveform $k$ at antenna element $n=p q$ and $(\cdot)^{H}$ is the Hermitian transpose operator.

The magnitude of the beampattern in the direction $(\theta, \phi)$ is given by

$$
\begin{aligned}
G(\theta, \phi) & =\int_{T} \mathbf{s}(t) \mathbf{s}^{H}(t) d t \\
& =\mathbf{a}^{H}(\theta, \phi) \mathbf{W}\left(\int_{T} \boldsymbol{\psi}(t) \psi^{*}(t) d t\right) \mathbf{W}^{H} \mathbf{a}(\theta, \phi) \\
& =\mathbf{a}^{H}(\theta, \phi) \mathbf{W} \mathbf{W}^{H} \mathbf{a}(\theta, \phi)=\left\|\mathbf{W}^{H} \mathbf{a}(\theta, \phi)\right\|^{2}
\end{aligned}
$$

where $\int_{T} \boldsymbol{\psi}(t) \boldsymbol{\psi}^{*}(t) d t=\mathbf{I}_{k}$, as $\boldsymbol{\psi}(t)$ are chosen to be orthogonal, $T$ is the period of a slow-time pulse, $\|\cdot\|$ is the Euclidean norm, and $(\cdot)^{*}$ is the complex conjugate.

The presence of $L$ targets in a Doppler-range bin following a Swerling II model results in a noisy $R \times 1$ receive array observation vector at a time $t$ and pulse $\tau$ which can be expressed as

$$
\mathbf{x}(t, \tau)=\mathbf{B} \boldsymbol{\Sigma}(\tau) \mathbf{A}^{H} \mathbf{W} \boldsymbol{\psi}(t)+\mathbf{z}(t, \tau)
$$

where $\mathbf{B} \triangleq\left[\mathbf{b}\left(\theta_{1}, \phi_{1}\right), \ldots, \mathbf{b}\left(\theta_{L}, \phi_{L}\right)\right], \mathbf{A} \triangleq\left[\mathbf{a}\left(\theta_{1}, \phi_{1}\right), \ldots\right.$ $\left.\ldots, \mathbf{a}\left(\theta_{L}, \phi_{L}\right)\right], \boldsymbol{\Sigma}(\tau) \triangleq \operatorname{diag}\left(\left[\beta_{1}(\tau), \ldots, \beta_{L}(\tau)\right]\right), \mathbf{z}(t, \tau)$ is an $R \times 1$ zero mean Gaussian random vector with covariance $\mathbf{Q}=\sigma^{2} \mathbf{I}_{R}$, and $\beta_{l}(\tau)$ is the complex radar reflection coefficient corresponding to the $l$-th target. The operator $\operatorname{diag}(\cdot)$ creates a diagonal matrix with entries equal to the elements of a vector. The receive array response vectors are $\mathbf{b}\left(\theta_{l}, \phi_{l}\right) \triangleq\left[e^{j 2 \pi \boldsymbol{\xi}\left[\gamma_{l}, \zeta_{l}\right]^{T}}\right]$ where $\boldsymbol{\xi}$ is an $R \times 2$ matrix containing the $x$ and $y$ coordinates of a receiver element $r$, $\gamma_{l} \triangleq \sin \theta_{l} \cos \phi_{l}$, and $\zeta_{l} \triangleq \sin \theta_{l} \sin \phi_{l}$. The receive antenna element coordinates in $\xi$ are defined relative to a reference element in terms of the carrier wavelength $\lambda_{c}$, but are otherwise arbitrary. Therefore, the first row of $\boldsymbol{\xi}$ is the zero row vector. The columns of the matrices $\mathbf{A}$ and $\mathbf{B}$ are the array response vectors for a target located at direction $\left(\theta_{l}, \phi_{l}\right)$ for the transmitter and receiver respectively.

Defining the $K \times N$ matrix $\boldsymbol{\Psi} \triangleq[\boldsymbol{\psi}(1), \ldots, \boldsymbol{\psi}(N)]$, where $N$ is the number of fast-time samples of the $K$ orthogonal waveforms, the result of the matched filter operation at the receiver over a slow-time pulse $\tau$ is expressed as

$$
\begin{aligned}
\mathbf{y}(\tau) & =\operatorname{vec}\left(\frac{1}{N} \mathbf{B} \boldsymbol{\Sigma}(\tau) \mathbf{A}^{H} \mathbf{W} \boldsymbol{\Psi} \boldsymbol{\Psi}^{H}+\frac{1}{N} \mathbf{z}(t, \tau) \boldsymbol{\Psi}^{H}\right) \\
& =\operatorname{vec}\left(\mathbf{B} \boldsymbol{\Sigma}(\tau) \mathbf{A}^{H} \mathbf{W}+\mathbf{V}(\tau)\right) \\
& =\left(\left(\mathbf{W}^{H} \mathbf{A}\right) \odot \mathbf{B}\right) \mathbf{c}(\boldsymbol{\Sigma}(\tau))+\mathbf{v}(\tau)
\end{aligned}
$$

where $\operatorname{vec}(\cdot)$ is the vectorization operator (which stacks the columns of a matrix on top of one another into a vector), $\odot$ is the column-wise Khatri-Rao product, $\mathbf{c}(\boldsymbol{\Sigma}(\tau))$ is a column vector consisting of the diagonal entries of $\boldsymbol{\Sigma}(\tau)$, and $\mathbf{v}(\tau) \triangleq$ $N^{-1} \operatorname{vec}\left(\mathbf{z}(t, \tau) \Psi^{H}\right)$.
The noisy virtual data vectors (5) have dimension of $K R \times 1$ and form a $K R \times I$ matrix

$$
\mathbf{Y} \triangleq[\mathbf{y}(1), \ldots, \mathbf{y}(I)]=\left(\left(\mathbf{W}^{H} \mathbf{A}\right) \odot \mathbf{B}\right) \mathbf{C}(\boldsymbol{\Sigma})+\mathbf{V}
$$

where $I$ is the number of slow-time pulses in a scan, $\mathbf{C}(\boldsymbol{\Sigma})$ has columns $\mathbf{c}(\boldsymbol{\Sigma}(\tau))$, and $\mathbf{V}$ has columns $\mathbf{v}(\tau)$.

In the following section, it will be shown that by placing a specific structure on $\mathbf{W}$ it is possible to guarantee multiple invariances within the received data while focussing energy from the transmitter into a desired sector. As such, not only do we benefit from energy focussing at the transmitter, but it is also possible to use search free DOA estimation techniques at the receiver without the requirement of a specific receiver array geometry.

\section{BEAMSPACE DESIGN}

The design of $\mathbf{W}$ is performed in two stages. First, a beamspace matrix $\mathbf{U}_{0}=\left[\mathbf{u}_{1}, \ldots, \mathbf{u}_{k}\right]$, with full column rank $K$, is designed over a spatial sector $\Theta=\left[\begin{array}{ll}\theta_{1} & \theta_{2}\end{array}\right]$ and $\Phi=\left[\begin{array}{ll}\phi_{1} & \phi_{2}\end{array}\right]$ using only the first $(P-1)$ rows and $(Q-1)$ columns of the transmit array. Then, a simple transformation is performed on $\mathbf{U}_{0}$ to produce beamforming matrices with identical beampatterns, but which correspond to different subarrays. The number of orthogonal waveforms $K$ is selected to be the number of dominant eigenvalues of the matrix

$$
\mathbf{D}(\theta, \phi) \triangleq \int_{\Theta} \int_{\Phi} \mathbf{a}(\theta, \phi) \mathbf{a}^{H}(\theta, \phi) d \theta d \phi .
$$

According to [13], $K \ll P Q$, given a sector area $\{\Theta, \Phi\}$.

The initial design of $\mathbf{U}_{0}$ can be stated as the following optimization problem

$$
\begin{aligned}
& \min _{\mathbf{u}_{1}, \ldots, \mathbf{u}_{K}} \max _{\theta, \phi}\left|G_{d}(\theta, \phi)-\sum_{k=1}^{K} \mathbf{u}_{k}^{H} \mathbf{a}(\theta, \phi) \mathbf{a}^{H}(\theta, \phi) \mathbf{u}_{k}\right| \\
& \text { s.t. } \sum_{k=1}^{K}\left|\mathbf{U}_{[j k]}\right|^{2}=\frac{E}{4 K P Q}, j \in\{1, \cdots,(P-1)(Q-1)\}
\end{aligned}
$$

where $G_{d}(\theta, \phi)$ is an ideal beampattern over the desired sector $\{\Theta, \Phi\}$. (9) is a non-convex quadratically-constrained quadratic optimization problem. It can be solved via a semidefinite programming relaxation approach, [13]- [15] by first introducing the new variables $\mathbf{X}_{k} \triangleq \mathbf{u}_{k} \mathbf{u}_{k}^{H}, k=1, \ldots, K$, and relaxing the rank constraint by requiring only that $\mathbf{X}_{k}$ be positive semidefinite. Then, using the technique of randomization a rank 1 solution can be extracted from the signal cross-correlation matrix $\mathbf{X}_{k}$. This will yield a beamforming matrix of dimension $(P-1)(Q-1) \times K$.

It is trivial to show that a matrix $\mathbf{U}_{0}^{\prime}$ of dimension $P Q \times$ $K$, with an identical beampattern to that of $\mathbf{U}_{0}$, can be constructed by placing zeros in the spots corresponding to the antenna elements which were omitted from the original design 
of $\mathbf{U}_{0}$. The matrix $\mathbf{U}_{0}^{\prime}$ then denotes a beamforming matrix where $K$ beams are transmitted from the first $P-1$ rows and $Q-1$ columns of a transmit array of dimension $P \times Q$. Given the shape of the transmit array, it is simple to show that by shifting the positions of the zeros in $\mathbf{U}_{0}^{\prime}$, the exact same beampattern can be achieved by subarrays containing the first $P-1$ rows and last $Q-1$ columns, the last $P-1$ rows and the first $Q-1$ columns, and finally the last $P-1$ and last $Q-1$ columns of the transmit array. These three matrices are denoted as $\mathbf{U}_{1}^{\prime}, \mathbf{U}_{2}^{\prime}$, and $\mathbf{U}_{3}^{\prime}$, respectively. With these matrices defined, it is easy to show that the following is true

$$
\begin{aligned}
\mathbf{a}^{H}(\theta, \phi) \mathbf{U}_{0}^{\prime} & =e^{j 2 \pi d_{x} \sin \theta \cos \phi}\left(\mathbf{a}^{H}(\theta, \phi) \mathbf{U}_{1}^{\prime}\right) \\
& =e^{j 2 \pi d_{y} \sin \theta \sin \phi}\left(\mathbf{a}^{H}(\theta, \phi) \mathbf{U}_{2}^{\prime}\right) \\
& =e^{j 2 \pi\left(d_{x} \sin \theta \cos \phi+d_{y} \sin \theta \sin \phi\right)}\left(\mathbf{a}^{H}(\theta, \phi) \mathbf{U}_{3}^{\prime}\right) .
\end{aligned}
$$

The beamforming matrix $\mathbf{W}$ is then defined as $\mathbf{W} \triangleq$ $\left[\mathbf{U}_{0}^{\prime}, \mathbf{U}_{1}^{\prime}, \mathbf{U}_{2}^{\prime}, \mathbf{U}_{3}^{\prime}\right]$ with an overall dimension of $P Q \times 4 K$. Clearly, in the original design problem, $K$ must be no larger than $P Q / 4$. It should be stated that the partially adaptive approach described in this section implies that only $(P-1)(Q-$ $1)$ elements operate at full power. This power loss, however, is a strictly decreasing function of transmit array size.

\section{SEARCH FREE DOA ESTIMATION}

Given the structure (10) imposed on the beamspace matrix $\mathbf{W}$, let us turn our attention to (5). Rewriting the noiseless matrix before vectorization allows a clear illustration of the effect of the proposed structure of $\mathbf{W}$ on DOA estimation. Specifically, we can write that

$$
\mathbf{B} \boldsymbol{\Sigma}(\tau) \mathbf{A}^{H} \mathbf{W}=\mathbf{B} \boldsymbol{\Gamma}
$$

where $\boldsymbol{\Gamma} \triangleq \boldsymbol{\Sigma}(\tau) \mathbf{A}^{H} \mathbf{W}$. The matrix $\boldsymbol{\Gamma}$ is the source signal matrix, and has dimension $L \times 4 K$. In the following, $\boldsymbol{\Gamma}_{0}=$ $\boldsymbol{\Sigma}(\tau) \mathbf{A}^{H} \mathbf{U}_{0}^{\prime}$ is the source signal matrix corresponding to $K$ beams emanated from the first $(P-1)$ rows and $(Q-1)$ columns of the transmit array. Using the relations (10) we define matrices $\boldsymbol{\Omega}_{i}, i \in\{0,1,2,3\}$ as the $L \times L$ diagonal matrices with the $l$-th diagonal entry of $\boldsymbol{\Omega}_{i}$ being the complex exponential in (10) which relates $\mathbf{a}^{H}(\theta, \phi) \mathbf{U}_{0}^{\prime}$ to $\mathbf{a}^{H}(\theta, \phi) \mathbf{U}_{i}^{\prime}$. The matrix $\Omega_{0}$ is obviously the identity matrix. Then (11) can be expressed as the following block partitioned matrix

$$
\begin{aligned}
\mathbf{B} \boldsymbol{\Gamma} & =\mathbf{B}\left[\boldsymbol{\Omega}_{0} \boldsymbol{\Gamma}_{0}\left|\boldsymbol{\Omega}_{1} \boldsymbol{\Gamma}_{0}\right| \boldsymbol{\Omega}_{2} \boldsymbol{\Gamma}_{0} \mid \boldsymbol{\Omega}_{3} \boldsymbol{\Gamma}_{0}\right] \\
& =\left[\mathbf{B} \boldsymbol{\Omega}_{0}\left|\mathbf{B} \boldsymbol{\Omega}_{1}\right| \cdots \mid \mathbf{B} \boldsymbol{\Omega}_{3}\right] \operatorname{bdiag}_{4}\left(\boldsymbol{\Gamma}_{0}\right)
\end{aligned}
$$

where $\operatorname{bdiag}_{m}(\cdot)$ takes a single matrix as an argument, and creates a block diagonal matrix whose $m$ blocks are equal to its argument. The matrix $\mathbf{B} \Omega_{0}$ is simply the receiver response matrix to $L$ targets. The virtual receiver response matrices $\mathbf{B} \Omega_{1}, \mathbf{B} \Omega_{2}$, and $\mathbf{B} \Omega_{3}$ are exactly the receiver response matrices to $L$ targets, for identical receive arrays that are linearly displaced from our actual receiver by $\left[d_{x}, 0\right],\left[0, d_{y}\right]$, and $\left[d_{x}, d_{y}\right]$, respectively. The source signal matrix $\boldsymbol{\Gamma}_{0}$ is a common factor for each. From (12) it is visible that the proposed structure for $\mathbf{W}$ enforces an algebraic structure on $\mathbf{Y}$ which can be exploited by search-free algorithms for DOA estimation, including, but not limited to, ESPRIT (Estimation of Signal Parameters through Rotational Invariance Techniques) [16], [17]. Further, it should be noted from (7) that the matrix $\mathbf{Y}$ has rank $L$ if the targets are incoherent. A proof of this statement is left for the journal version.

Matrix $\mathbf{Y}$ has dimension $4 R K \times I$. After defining the matrix selection operator $\mathbf{F}_{j}(\cdot)$ which selects the $(j M / 4+1)-$ $M / 4(j+1)$ rows from an arbitrary matrix with $M$ rows, where $j \in\{0,1,2,3\}, \mathbf{Y}$ and a new matrix $\mathbf{Y}^{\prime}$ can be expressed as

$$
\mathbf{Y}=\left[\begin{array}{l}
\mathbf{F}_{0}(\mathbf{Y}) \\
\mathbf{F}_{1}(\mathbf{Y}) \\
\mathbf{F}_{2}(\mathbf{Y}) \\
\mathbf{F}_{3}(\mathbf{Y})
\end{array}\right], \mathbf{Y}^{\prime}=\left[\begin{array}{l}
\mathbf{F}_{0}(\mathbf{Y}) \\
\mathbf{F}_{2}(\mathbf{Y}) \\
\mathbf{F}_{1}(\mathbf{Y}) \\
\mathbf{F}_{3}(\mathbf{Y})
\end{array}\right]
$$

Forming the cross correlation matrices $\mathbf{R}_{\mathbf{Y}}=I^{-1} \mathbf{Y} \mathbf{Y}^{H}$ and $\mathbf{R}_{\mathbf{Y}^{\prime}}=I^{-1} \mathbf{Y}^{\prime} \mathbf{Y}^{\prime H}$, and performing ESPRIT on both will yield a vector of $L$ phase arguments which are directly proportional to $\zeta_{l}$ and $\gamma_{l}$. Defining a complex number $z_{l}=$ $\gamma_{l}+j \zeta_{l}$ the angle estimates are given by $\phi_{l}=\arctan \left(\zeta_{l} / \gamma_{l}\right)$, and $\theta_{l}=\left|z_{l}\right|$.

\section{SIMULATIONS}

To evaluate the DOA estimation performance of the proposed method, we consider both the root mean square error (RMSE) of the estimates, and the probability of resolving two closely located targets. The RMSE is calculated as

$$
R M S E=\sqrt{\frac{1}{M} \sum_{m=1}^{M}\left(\frac{1}{L} \sum_{l=1}^{L}\left\|\hat{\rho}_{l}-\rho_{l}\right\|^{2}\right)}
$$

where $M$ is the number of Monte Carlo trials, $\rho_{l}=\left[\begin{array}{ll}\theta_{l} & \phi_{l}\end{array}\right]$ are the target locations, and $\hat{\rho}_{l}=\left[\hat{\theta}_{l} \hat{\phi}_{l}\right]$ are their estimates. If $\left\|\hat{\rho}_{l}-\rho_{l}\right\| \leq\left\|\rho_{1}-\rho_{2}\right\| / 2, l \in\{1,2\}$ then the two targets are said to be resolved.

We compare the performance of the proposed method with that of MIMO radar using uniform transmit power, and full waveform diversity (hereafter referred to as conventional MIMO radar). Both methods are tested using the same system configuration of an $11 \times 11$ URA with antenna element spacing $\lambda_{c} / 2$ acting as the transmitter, and a planar array with randomized geometry and 8 antenna elements acting as the receiver. That is, the $\mathrm{x}$ and $\mathrm{y}$ coordinates of each receive antenna element are randomly and independently drawn from a uniform distribution $\mathcal{U}\left[0,4 \lambda_{c}\right]$. Both methods use exponential harmonic waveforms. The proposed method 
uses 32 exponential harmonic waveforms, transmitted in 4 groups of 8 waveforms each. The conventional MIMO radar, having full waveform diversity, transmits 121 exponential harmonic waveforms. The beampattern for the proposed method is designed over a desired sector of $\Theta=\left[30^{\circ} 50^{\circ}\right]$ and $\Phi=\left[100^{\circ} 120^{\circ}\right]$.

While designing the transmit beamspace matrix, we allowed a transition region of $10^{\circ}$ width around the mainlobe in the elevation domain, and $20^{\circ}$ in the azimuthal domain. Otherwise, the side-lobes of the beampattern are forced to be as low as possible, while matching the passband of the designed beampattern as closely to that of the desired one as possible. We then extract the beamspace matrix $\mathbf{U}_{0}$ by selecting the best of 5000 random candidate matrices. The beamspace matrix $\mathbf{W}$ is then constructed. Figs. 1 and 2 show the beampattern while looking directly at the elevation and azimuthal aspect, respectively.

To compare first the RMSE and second, the probability of target resolution for the two methods tested, two separate scenarios are considered. In the first, a single target following a Swerling II model is placed at $\rho=\left[47^{\circ} 106^{\circ}\right]$ In the second, two targets following a Swerling II model are placed at $\rho_{1}=$ $\left[47^{\circ} 106^{\circ}\right]$ and $\rho_{2}=\left[48^{\circ} 107^{\circ}\right]$. Figs. 3 and 4 shows the comparison between the proposed methods tested over 500 Monte Carlo trials as a function of signal to noise ratio (SNR), which varies from -30 to $30 \mathrm{~dB}$ in $5 \mathrm{~dB}$ increments. The proposed ESPRIT-based algorithm is used to extract the angle estimates.

As can be seen in Figs. 3 and 4, the proposed method enjoys significant performance benefits compared to the conventional MIMO radar in both RMSE and probability of target resolution. The most noticeable difference lies in the probability of target resolution, where the gap between the pro-

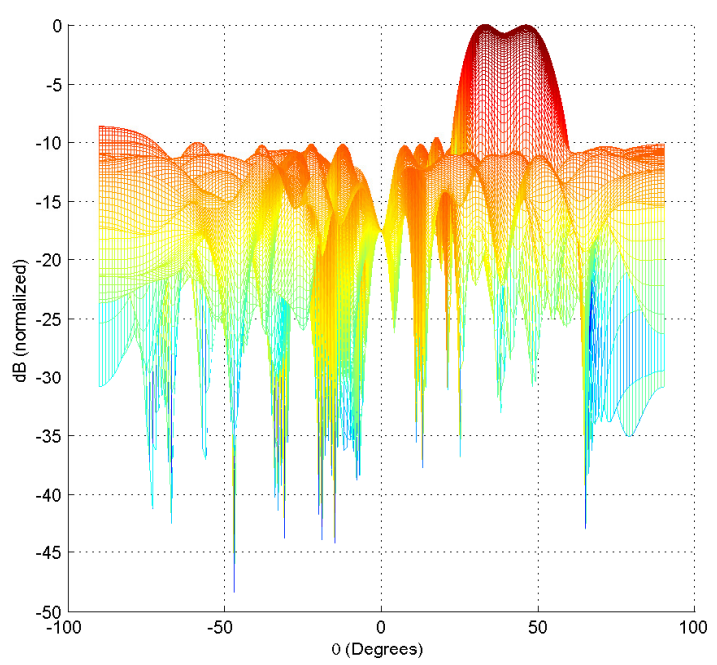

Fig. 1. Transmit Beampattern: elevation aspect.

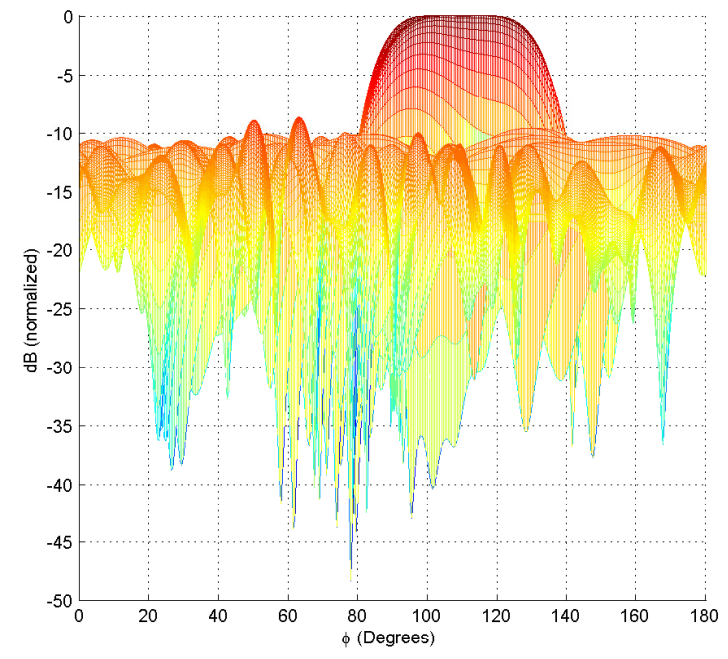

Fig. 2. Transmit beampattern: azimuthal Aspect.

posed method and the conventional MIMO radar is as wide as $10 \mathrm{~dB}$. In terms of RMSE, as SNR climbs above $10 \mathrm{~dB}$, the gap between the two methods begins to close. However, while the RMSE performance of the conventional MIMO radar is close to that of the proposed method at high SNR, the proposed method still benefits from a significantly lower computational complexity. The calculations required per iteration for the conventional MIMO radar are $\mathcal{O}\left((P Q R)^{3}\right)$ while the calculations required for proposed method are $\mathcal{O}\left((4 K R)^{3}\right)$. With the configuration used in this example, the conventional MIMO radar requires about 50 times the calculations of the proposed method.

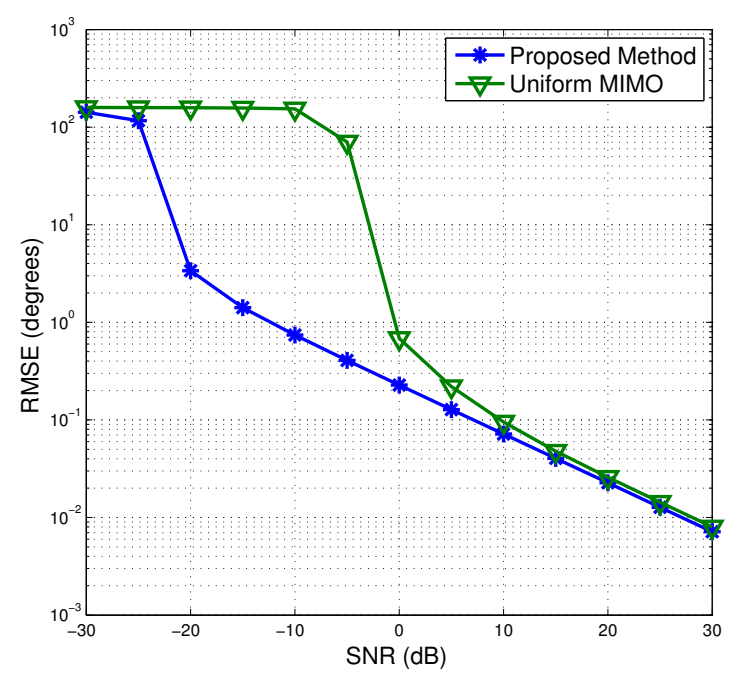

Fig. 3. RMSE over 500 trials, from -30 to $30 \mathrm{~dB}$. 


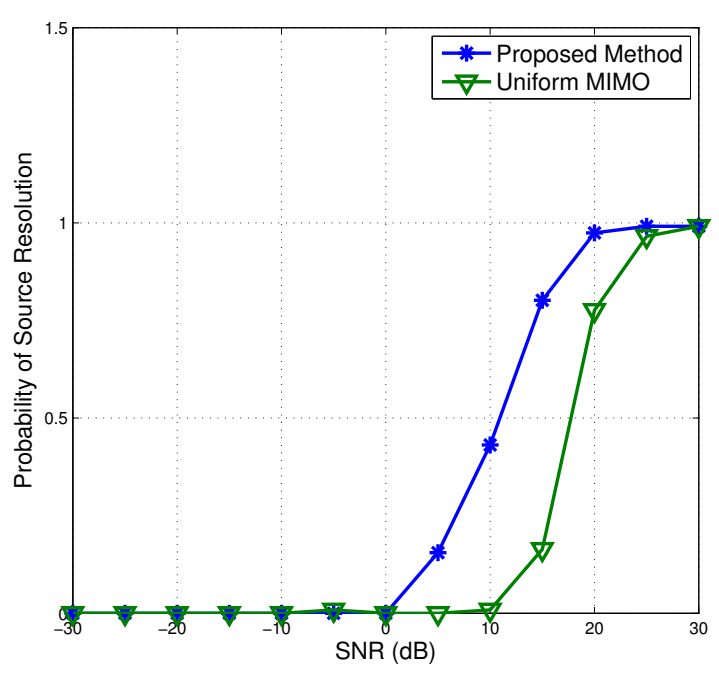

Fig. 4. Probability of Source Resolution, 500 trials, from -30 to $30 \mathrm{~dB}$.

\section{CONCLUSION}

The problem of 2D transmit beamforming for planar arrays with the specific goal of creating a data set at the receiver with multiple invariances independently of the receive array geometry has been considered. A partially adaptive approach which creates a set of identical beams that are transmitted from different subarrays has been used. Rotational invariance between the beams has been shown to allow for existence of search free DOA estimation methods of both azimuth and elevation angles. RMSE and probability of target resolution have been used to compare the proposed method with the conventional MIMO radar. The proposed method has been shown to have a significant advantage in accuracy and resolution, while having the additional benefit of substantially reduced computational complexity.

\section{REFERENCES}

[1] J. Li and P. Stoica, MIMO Radar Signal Processing. New Jersy: Wiley, 2009.

[2] J. Li and P. Stoica, "MIMO radar with colocated antennas," IEEE Signal Process. Magaz., vol. 24, pp. 106-114, Sept. 2007.

[3] A. Haimovich, R. Blum, and L. Cimini, "MIMO radar with widely separated antennas," IEEE Signal Process. Magaz., vol. 25, pp. 116-129, Jan. 2008.

[4] C.-Y. Chen and P.P. Vaidyanathan, "MIMO radar space-time adaptive processing using prolate spheroidal waveform functions," IEEE Trans. Signal Process., vol. 56, pp. 623-635, Feb. 2008.
[5] C. Chen, P. P. Vaidyanathan, "MIMO radar ambiguity properties and optimization using frequency-hopping waveforms," IEEE Trans. Signal Process., vol. 56, pp. 5926-5936, Dec. 2008.

[6] E. Fishler, A. Haimovich, R. Blum, L. Cimini, D. Chizhik, and R. Valenzuela, "Spatial diversity in radars: Models and detection performance," IEEE Trans. Signal Process., vol. 54, pp. 823-838, Mar. 2006.

[7] A. Hassanien, S. A. Vorobyov, and A. B. Gershman, "Moving target parameters estimation in non-coherent MIMO radar systems," IEEE Trans. Signal Process., vol. 60, pp. 2354-2361, May 2012.

[8] A. Hassanien and S. A. Vorobyov, "Phased-MIMO radar: A tradeoff between phased-array and MIMO radars," IEEE Trans. Signal Process., vol. 58, pp. 3137-3151, June 2010.

[9] A. Hassanien and S. A. Vorobyov, "Transmit energy focusing for DOA estimation in MIMO radar with colocated antennas," IEEE Trans. Signal Process., vol. 59, pp. 2669-2682, June 2011.

[10] D. Wilcox and M. Sellathurai, "On MIMO radar subarrayed transmit beamforming," IEEE Trans. Signal Process., vol. 60, no. 4, pp. 2076-2081, Apr. 2012.

[11] A. Hassanien, S. A. Vorobyov, Y.-S. Yoon, and J.-Y. Park, "Two-stage based design for phased-MIMO radar with improved coherent transmit processing gain," in Proc. SPAWC, Toronto, Canada, June 2014, pp. 45-49.

[12] A. Hassanien, S. A. Vorobyov, Y.-S. Yoon, and J.-Y. Park, "Root-MUSIC-based source localization using transmit array interpolation in MIMO radar with arbitrary planar array," in Proc. IEEE CAMSAP'2013, The Friendly Island, Saint Martin, Dec. 2013, pp. 396-399.

[13] A. Hassanien, M. W. Morency, A. Khabbazibasmenj, S. A. Vorobyov, J.-Y. Park, and S.-J. Kim, "Two-dimentional transmit beamforming for MIMO radar with sparse symmetric arrays," in Proc. IEEE Radar Conf., Ottawa, ON, Canada, Apr.-May 2013, pp. 1-5.

[14] A. Khabbazibasmenj, A. Hassanien, S. A. Vorobyov, M. W. Morency, "Efficient transmit beamspace design for search-free based DOA estimation in MIMO radar," IEEE Trans. Signal Process., vol. 62, no. 6, pp. 1490-1500, Mar. 2014.

[15] A. Khabbazibasmenj, S. A. Vorobyov, A. Hassanien, and M. W. Morency, "Transmit beamspace design for direction finding in colocated MIMO radar with arbitrary receive array and even number of waveforms," in Proc 46th Annual Asilomar Conf., Pacific Grove, California, USA, Nov. 2012, pp. 1307-1311.

[16] A. Paulraj, R. Roy, and T. Khalaith, "Estimation of signal parameters via rotational invariance techniques- Esprit," in Proc. 19th Annual Asilomar Conf., Pacific Grove, California, USA, Nov. 1985, pp. 83-89.

[17] N.D. Sidiropoulos, R. Bro, and G.B. Giannakis, "Parallel factor analysis in sensor array processing," IEEE Trans. Signal Process., vol. 48, pp. 2377-2388, Aug. 2000. 